Supplemento n. 3

Giornale di Tecniche

Nefrologiche \& Dialitiche

Anno XIV ${ }^{\circ} 3$

(C) Wichtig Editore 2002

\title{
Cateteri venosi centrali e adeguatezza dialitica
}

\author{
Salvatore Mandolfo
}

U.O. di Nefrologia e Dialisi, Azienda Ospedaliera della Provincia di Lodi, Lodi

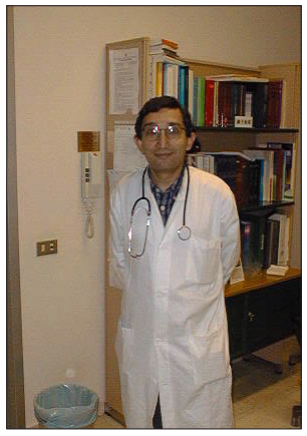

L' impiego del catetere venoso centrale (CVC), quale accesso vascolare per l'emodialisi, rappresenta un fenomeno in continua crescita. Come sottolineato dalla recente inchiesta DOPPS, nei cinque paesi europei esaminati, il $31 \%$ dei pazienti inizia un trattamento dialitico con un CVC e il $7 \%$ ha quale accesso definitivo un CVC permanente (CVCp). I motivi che possono spiegare questo esteso impiego del CVC sono rappresentati da un ricorso tardivo al nefrologo, da una inadeguata programmazione di un accesso definitivo, dalle condizioni sempre più compromesse dell'albero vascolare dei pazienti che sono sottoposti a trattamento dialitico e non ultimo dal proprio contesto operativo (per esempio, disponibilità del chirurgo vascolare).

Lo scopo del presente lavoro è di considerare il problema dell'adeguatezza dialitica nei pazienti in emodia- lisi con un CVCp.

Quando si parla di accessi vascolari e adeguatezza dialitica, i problemi principali da affrontare riguardano il ricircolo, il flusso ematico effettivo e la portata dell'accesso.

\section{Ricircolo}

Il ricircolo è la quota di sangue depurato dal circuito extra-corporeo che ritorna al filtro senza passare attraverso il sistema dei capillari del circolo sistemico. Dal punto di vista clinico assume particolare importanza in quanto riduce la rimozione dei soluti e pertanto determina una diminuzione dell'efficienza dialitica. Sono stati dimostrati due tipi di ricircolo: il ricircolo dell'accesso vascolare e il ricircolo cardio-polmonare. Nel CVC, contrariamente alla fistola, non esiste la quota cardio-polmonare, mentre assume particolare importanza il "design" e in particolare la distanza fra i due lumi del catetere. In genere nei CVCp, quali il PermCathTM, l'Ash-SplitTM, l'OptiflowTM, il VasCathTM se correttamente utilizzati, i valori di ricircolo sono inferiori al $3 \%$ e pertanto di scarso significato clinico in termini di efficienza dialitica. Discorso a parte va fatto per il catetere di TesioTM, il quale essendo costituito da due cannule distinte e separate, è importante al momento dell'impianto, posizionare le punte dei due cateteri distanti fra loro di circa quattro centimetri.

Il problema del ricircolo con i CVCp assume importanza in termini di efficienza dialitica qualora il lume arterioso venga utilizzato come rientro venoso e il lume venoso come aspirazione. Questa è una evenienza abbastanza frequente nelle nostre sale dialisi e può essere il risultato di una necessità clinica (la linea arteriosa non consente flussi adeguati) oppure di un errore tecnico da parte del personale. Twardowski et al nel 1993 hanno dimostrato, su $18 \mathrm{CVC}$ alti (succlavia e giugulari) che se l'inversione è dovuta a necessità cliniche, e quindi il $\mathrm{CVC}$ è mal funzionante il ricircolo sarà intorno al $7 \%$, mentre se è dovuta a un errore umano (CVC ben funzionante) il ricircolo sarà oltre il 12 $\%$. Tale esperienza è stata recentemente confermata da Level et al, i quali hanno documentato un ricircolo del $12 \pm 9 \%$ in CVCp (Tesio) con linee 
invertite. Un altro aspetto importante riguarda la sede del CVC. In tal senso Leblanc et al, su 50 CVC (succlavia e femorale) relativi a 38 pazienti, hanno dimostrato che, nel caso si utilizzi la vena femorale, e quindi un approccio venoso basso, a parità di lunghezza il CVC determina un ricircolo quattro volte superiore a quello che si ottiene in succlavia. Lo stesso autore ha documentato a livello femorale, con cateteri di lunghezza di $15 \mathrm{~cm}$, un ricircolo due volte superiore nei confronti di cateteri di lunghezza maggiore di $19 \mathrm{~cm}$.

Alla luce di tali esperienze può essere suggerito di utilizzare cateteri almeno di 19 centimetri quando si utilizza la sede femorale e di aumentare i tempi di trattamento dialitico, di almeno 30 minuti, qualora si utilizzi il lume venoso come aspirazione per necessità cliniche.

\section{Portata di un CVC}

Diversi sono i fattori che spiegano la differenza fra la portata di un CVC e quella di una FAV (nativa o con vasi protesici). La velocità di flusso del vaso utilizzato è sicuramente la principale e in tale ottica il CVC è notevolmente penalizzato. Nel caso di un CVC, la portata viene descritta attraverso la legge di Hagen-Poiseuille. Dipende in modo diretto dalla sezione interna e dalla differenza di pressione tra l'ingresso e l'uscita della cannula, in modo inverso dalla viscosità ematica e dalla lunghezza della cannula. Per quanto riguarda la sezione, $\mathrm{i}$ CVCp presentano un diametro interno di $2 \mathrm{~mm}$ e quindi maggiore a quello dei comuni aghi fistola per dialisi. La caduta di pressione, nei CVC, come dimostrato in modo sperimentale da Pollaschegg, avviene nel punto di connessione fra la cannula e le linee del circuito extracorporeo e particolare importanza assume il "design" del CVCp. La viscosità ematica, si correla in modo esponenziale con l'ematocrito. In tal senso, l'utilizzo in modo esteso dell'eritropoietina e in particolare dei "target" di ematocrito sempre più elevati, possono influire sulla resa di un CVCp specie se vengono superati i valori di $40 \%$ nella parte finale del trattamento dialitico a seguito dell'emoconcentrazione. Per quanto riguarda la lunghezza, in teoria, più un catetere è corto e migliore sarà la sua portata. Tale aspetto tuttavia si scontra con la realtà clinica, in quanto per motivi anatomici, per ottenere dei flussi adeguati in femorale, è necessario utilizzare cateteri di almeno $20 \mathrm{~cm}$ $\mathrm{e}$ in giugulare molte volte è necessario posizionare la punta del CVC in prossimità dell'atrio.

\section{Flusso ematico effettivo}

Il flusso ematico rappresenta uno degli aspetti fondamentali per ottenere una dialisi adeguata, in quanto da esso dipende in modo esponenziale la clearance del filtro. A titolo di esempio, per un paziente di media taglia (60-70 kg), se vogliamo mantenere i tempi di trattamento intorno alle quattro ore, si rende necessario un $\mathrm{Qb}$ di almeno $250 \mathrm{mil} / \mathrm{min}$. Il problema dei flussi con i CVC diventa ancora più importante se consideriamo il flusso ematico effettivo, in altre parole la quota di sangue che effettivamente raggiunge il filtro. Quest'ultima dipende, in modo diretto dalla portata dell'accesso, in modo inverso dalla quota di ricircolo e dalla pressione di aspirazione del circuito extra corporeo. Tale problema, già noto per le FAV, diventa ancora più importante per il CVC, in quanto quest'ultimo presenta delle portate inferiori alla fistola e per consentire flussi ematici intorno a $300 \mathrm{ml} / \mathrm{min}$, è necessario ricorrere a pressioni di aspirazione più elevate.

I numerosi cateteri a doppio lume presenti sul mercato, in base a quanto dichiarato dalle ditte, sono in grado di garantire flussi ematici oltre i 400 $\mathrm{ml} / \mathrm{min}$ con pressioni di aspirazione tra i 150 ed i $200 \mathrm{mmHg}$. Tali dati erano stati confermati dalle prime esperienze cliniche, specie americane, ove nell' $80-90 \%$ dei pazienti veniva riportata la possibilità di ottenere dei flussi ematici superiori a $400 \mathrm{ml} / \mathrm{min}$. Tuttavia nella nostra pratica clinica raramente è stato possibile ottenere tali valori. Per confermare tale impressione, abbiamo pertanto condotto uno studio in collaborazione con i Centri dialisi di Pavia (FSM), Voghera, Brescia e Cremona. Su 57 pazienti con CVCp, i risultati hanno mostrato un flusso medio pari a $269 \pm 47$ $\mathrm{ml} / \mathrm{min}$, e nel $38 \%$ dei pazienti il flusso era inferiore a $250 \mathrm{ml} / \mathrm{min}$. Questo ha comportato che per pazienti di peso corporeo maggiore di $60 \mathrm{~kg}$, è stato necessario aumentare i tempi di trattamento per ottenere un valore di $\mathrm{Kt} / \mathrm{V}=1.2 \mathrm{e}$ in tre casi (pazienti di oltre $75 \mathrm{~kg}$ ), sebbene i tempi di dialisi fossero stati aumentati a 5 ore, non è stato possibile ottenere un valore adeguato di Kt/V. Recentemente i nostri dati sono stati confermati da una grossa casistica americana (184 CVCp relativi a 132 pazienti), durata 21 mesi, ove è emerso che il flusso medio è pari a $281 \mathrm{ml} / \mathrm{min}$ ed è stato necessario rimuovere $19 \mathrm{CVCp}$ per flussi inadeguati.

\section{Cause di flusso ematico inadeguato}

Per un CVCp si definisce adeguato, un flusso ematico superiore ai 250 $\mathrm{ml} / \mathrm{min}$ in corso di trattamento emodialitico. Un flusso inadeguato predispone a una maggiore incidenza di infezioni oltre che a un quadro di sottodialisi.

In accordo a Leblanc M. et al, le cause di flusso ematico inadeguato, possono essere distinte in precoci e tardive. Le cause precoci sono quasi sempre secondarie a problemi meccanici, e quindi a problemi di posizionamento, quali una piegatura o a una inadeguata sede della punta, tuttavia non si può escludere a priori un precoce deposito di fibrina. Le cause tardive sono invece dovute a problemi di gestione e quasi sempre a problemi di tipo trombotico. In tal senso occorre ricordare che il trombo può essere all'interno del lume, all'interno del vaso venoso sia a monte che a valle del CVCp, ma molte volte è rappresentato da un manicotto di fibrina intorno al CVCp. Molti CVCp, specie dopo l'impianto presentano un avvio difficile, e in mancanza di problemi meccanici o depositi di fibrina, si ipotizza che il distretto venoso che ospita il 
catetere debba, in un certo senso, adattarsi alla nuova presenza. L'esperienza clinica ci insegna inoltre che molti CVCp presentano dei flussi inadeguati, sebbene posizionati in modo corretto e in assenza di apparenti fenomeni trombotici. Al fine di poter spiegare questo fenomeno, nello studio multicentrico prima riportato, $\mathrm{i}$ pazienti sono stati divisi in 2 gruppi in funzione del $\mathrm{Qb}$, il primo con $\mathrm{Qb}$ $<280 \mathrm{ml} / \mathrm{min}$, il secondo con $\mathrm{Qb}>280$ $\mathrm{ml} / \mathrm{min}$. Fra i due gruppi nessuna differenza è emersa negli esami ematochimici (viscosità) e nei valori di pressione arteriosa del paziente, a inizio e fine dialisi. La terapia con antiaggreganti non è sembrata influenzare l'efficienza del CVC. I pazienti del secondo gruppo avevano avuto un minor numero di infezioni. Il dato più significativo era tuttavia dato dalla sede della punta del CVCp, in particolare nei pazienti ove la punta era localizzata in prossimità dell'atrio destro, i flussi erano significativamente più elevati $(287 \pm 20 \mathrm{ml} / \mathrm{min})$ nei confronti dei pazienti con punta del CVCp posizionata nella vena cava superiore $(268 \pm 39 \mathrm{ml} / \mathrm{min})$. I pazienti con CVCp situato in vena femorale presentavano i flussi peggiori (244 $\pm 27 \mathrm{ml} / \mathrm{min})$.

\section{Considerazioni finali}

Il posizionamento di un CVCp per emodialisi deve rappresentare un momento di riflessione del nefrologo, il quale da una parte deve conoscere $\mathrm{i}$ possibili rischi iatrogeni legati al posizionamento, dall'altra deve saper gestire le possibili complicanze, quali le infezioni e le trombosi. Un'ulteriore problematica legata al CVCp può essere rappresentata dai flussi ematici effettivi inferiori rispetto a una FAV, ciò comporta una ridotta efficienza dialitica e predispone il paziente a un quadro di sottodialisi.

nefrodialisilodi@pmp.it 